\title{
Ongoing Epidemiological Changes in Hand, Foot, and Mouth Disease Following the Introduction of Enterovirus 71 Vaccines in Yunnan Province, China, 2008-2019
}

\author{
Li Jiang \\ Kunming Children's Hospital \\ Hongchao Jiang \\ Kunming Children's Hospital \\ Xin Tian \\ Kunming Children's Hospital \\ Xueshan Xia \\ Kunming University of Science and Technology \\ Tian Huang ( $\sim 99600694 @ q q . c o m$ ) \\ Yunnan Provincial Center for Disease Control and Prevention
}

\section{Research Article}

Keywords: Hand, foot, and mouth disease, Enterovirus 71 vaccine, Epidemiology

Posted Date: March 11th, 2021

DOI: https://doi.org/10.21203/rs.3.rs-276288/v1

License: (c) (i) This work is licensed under a Creative Commons Attribution 4.0 International License. Read Full License

Version of Record: A version of this preprint was published at BMC Infectious Diseases on August 4th, 2021. See the published version at https://doi.org/10.1186/s12879-021-06462-4. 


\section{Abstract}

\section{Background:}

Since 2016, enterovirus 71 (EV71) vaccines have been approved for market entry, and litter is known about how the epidemiology of hand, foot, and mouth disease (HFMD) has been affected by the introduction of the vaccines in Yunnan Province. The study describes the ongoing epidemiological changes in HFMD following the introduction of EV71 vaccination in Yunnan.

\section{Methods:}

Surveillance data collected between 2008 and 2019 were analyzed to produce EV71 vaccination coverage, epidemiological distribution on cases, and etiologic composition as well as to compare these characteristics before and after EV71 vaccination.

\section{Results:}

A total of 1,653,533 people received EV71 vaccines from 2016 through 2019 in Yunnan. The cumulative EV71 vaccination coverage rate was $68.14 \%$. After the introduction of EV71 vaccines, the overall incidence of HFMD increased and reached over 200 cases per 100,000 population-years in 2018 and 2019. However, the case severity and case fatality rate decreased and remained lower than $1 \%$ and $0.005 \%$ after 2016, respectively. EV71-associated mild, severe and fatal cases sharply decreased. The predominant viral serotype changed to non-EV71/non-CV-A16 enteroviruses which were detected across the whole province.

\section{Conclusions:}

EV71 vaccination helped to reduce severe HFMD. However, other enteroviruses became the predominant strain and let to a higher incidence. Expanding EV71 vaccination and strengthening laboratory-based surveillance could further decrease the burden of severe HFMD and reveal the existence of emerging enteroviruses.

\section{Introduction}

Hand, foot, and mouth disease (HFMD) is a viral illness mostly seen among children under 5 years old in the West Pacific, that causes mild symptoms including fever, erythra, vesiculation, and inappetence. However, some symptoms are severe and involve neurological complications and can even lead to death [1]. HFMD has been categorized as a class $C$ notifiable disease in China since May 2, 2008. By the end of 2015, over 13 million HFMD cases were reported, including 123,261 severe cases and 3,322 deaths in mainland China. Laboratory surveillance results showed that the causes of HFMD are enterovirus 71 (EV71) and coxsackievirus A16 (CV-A16) in most cases. Moreover, EV71 is the most frequently identified serotype among both severe and fatal cases [2]. 
Currently there is no specific antiviral treatment for HFMD. Three inactivated monovalent EV71 vaccines from Beijing Vigoo Biological Co., Ltd. (Vigoo), Sinovac Biotech Co., Ltd. (Sinovac), and the Institute of Medical Biology, Chinese Academy of Medical Sciences (Kunming Institute) were recently licensed in China, which showed high efficacy (90.0-97.4\%) against EV71-associated HFMD but no cross-protection against HFMD caused by CV-A16 or other serotypes in children [3-5].

Since 2016, EV71 vaccines have been introduced in Yunnan Province, China. The vaccine is voluntary and self-paid. It was recommended that children aged of 6 months to 5 years old receive the first dose, as well as a second dose on day 28 . However, the impact of this new vaccine on the epidemiology of HFMD is currently unknown after the introduction of EV71 vaccines in the province. Thus, we accessed the surveillance data of HFMD reported from 2008 through 2019 in Yunnan in an attempt to describe the epidemiological features of HFMD patients following the introduction of EV71 vaccines.

\section{Method}

\section{Study Area}

Yunnan is a province of China located in the most southwest of the country. It spans approximately 394,000 square kilometers and has a population of 48 million (2019). We divided the province into 4 regions, including central, northeastern, northwestern, and southern areas, according to proximity, culture, and socioeconomic status. More than half of the population lives in the central and northeastern parts of the province, which accounts for $30 \%$ of the total area of the province.

\section{Data sources}

Data on HFMD cases from 1 January 2008 to 31 December 2019 were obtained from the National Surveillance of Notifiable Infectious Disease Programme (NSNIDP), which was constructed by the China Centers for Disease Control and Prevention (China CDC). HFMD was required as a notifiable infectious disease to be reported to the NSNIDP since May 2, 2008. Basic demographic and diagnostic information and etiological results of HFMD cases and population by age group were collected via the NSNIDP. The vaccinated population in Yunnan was extracted from the Immunization Planning Information Management System (IPIMS) at the Yunnan CDC. When registration in the NSNIDP and IPIMS, written informed consent was obtained from each adult patient and legally authorized representatives of patient who was under 18 year of age. Data obtained from the NSNIDP and the IPIMS were anonymized and did not include any personal data.

According to HFMD diagnostic criteria, cases were categorized as severe when they presented more than one of the following complications: encephalitis, aseptic meningitis, or acute flaccid paralysis, pulmonary edema, pulmonary hemorrhage, or myocarditis, otherwise as mild. 
Virological surveillance was conducted by local CDC. Specimens were collected from cases and sent to laboratories for coxsackie virus A 16 (CV-A16) and enterovirus 71 (EV71) and other enterovirus nucleic acid testing by polymerase chain reaction or virus isolation. All methods were performed in accordance with the relevant guidelines and regulations that have been described in a previous study [6].

\section{Statistical analysis}

All data in this study were transferred into the R program for data exploration and analysis. As individual vaccination data were lacking, the annual vaccination coverage rate was estimated as the proportion of EV71 vaccinated people among the population of newborn children each year in Yunnan. The cumulative EV71 vaccination coverage rate from 2016 through 2019 was estimated as the proportion of total EV71 vaccinated people among children born from 2016 to 2019 in Yunnan. The incidence rate of HFMD was calculated by dividing the number of all reported HFMD cases by the total person-year in the corresponding time. The case severity rate and case fatality rate were identified as the proportion of severe cases and fatal cases among all reported cases, respectively. The seasonal pattern was described by year and month. The geographic distributions were marked by the four regions, including the central, northeastern, northwestern, and southern areas of Yunnan. The demographic characteristics, including gender and age, were presented as percentages and medians by pie and box graphs, respectively. Annual virological surveillance results were presented in percent bar graphs to explore the viral serotype epidemic cycle.

Furthermore, the EV71 vaccine was launched in Yunnan Province in 2016. 2017-2019 as the implementation period of the EV71 vaccination program to reflect the changes in HFMD epidemiology post-EV71 vaccine introduction. 2013-2015, as the baseline years before EV71 vaccination, was identified as pre-EV71 vaccine introduction. Epidemiological information on patient demographics, clinical classifications and viral serotypes was compared between before and after EV71 vaccine introduction. The significance of differences was initially assessed with the $X^{2}$ test and rank sum test.

\section{Results}

\section{EV71 Vaccination rate}

A total of 1,653,533 people received the EV71 vaccine from 2016 through 2019 in Yunnan. The annual vaccination rate showed an upward trend during the study period and was steady at $80 \%$ above in 2018 and 2019. The cumulative EV71 vaccination coverage rate among eligible children (born from 2016 to 2019) was $68.14 \%$ between 2016 and 2019 (Table no. 1).

Table 1

The EV71 vaccination coverage rate in Yunnan Province, 2016-2019 


\begin{tabular}{|llll|}
\hline Year & Number of people vaccinated & Population & $\begin{array}{l}\text { Coverage rate } \\
(\%)\end{array}$ \\
\hline 2016 & 192700 & 609001 & 31.64 \\
\hline 2017 & 455630 & 594103 & 76.69 \\
\hline 2018 & 471911 & 563405 & 83.76 \\
\hline 2019 & 533292 & 660311 & 80.76 \\
\hline Cumulative & 1653533 & 2426820 & 68.14 \\
\hline
\end{tabular}

\section{Incidence, Severity, and Fatality Rates of HFMD}

From 2008 through 2019, a total of 763,863 HFMD cases were reported in the NNIDRS. Of these cases, 9,928 cases were diagnosed as severe and included 144 deaths. The incidence of reported HFMD cases showed an upward trend since the initiation of surveillance in 2008 and reached a peak of over 200 cases per 100,000 population-years in 2018 (Fig 1 A). Both the case severity rate (Fig 1 B) and case fatality rate (Fig 1 C) presented large fluctuations during 2008-2015. However, the two rates continued to show downward trends and remained at lower levels after 2016.

\section{Seasonal Pattern}

HFMD was prevalent throughout the year in Yunnan, there was a single peak in May in 2008 and 2009. Since 2010, annual epidemic waves with a major peak in the early summer (May) followed by a smaller peak in autumn (November) were observed. However, the scales of the autumn peaks in 2013, 2015, 2017, and 2019 were smaller than those in the corresponding previous year (Fig 2).

\section{Geographic Distribution}

Of all cases reported, 324,822 (42.52\%), 200,107 (26.20\%), 160,773 (21.05\%), and 78,161 (10.23\%) cases were diagnosed from the central, southern, northwestern, and northeastern regions of the province during the surveillance period, respectively. Similar patterns of case geographic distribution were presented in each year. (Fig 3).

\section{Characteristics of Age and Gender}

There were 446,942 male and 316,921 female cases, as indicated by a male-to-female ratio of 1.41:1 among the reported cases. The proportions of case stratified by sex in each year are depicted by a pie graph (Fig 4 A). Males accounting for more than half of the cases were observed in each surveillance 
year. The distributions of age are shown by year and sex using box plots (Fig 4 B). The median age ranged from 2.3 to 2.9 years during the surveillance years.

\section{Etiologic Composition}

Laboratory tests confirmed 65,319 cases during 2008-2019, including 61,120 mild cases and 4,199 severe cases, of which 75 were fatalities. Among the laboratory-confirmed cases, 20,475 (31.35\%), 22,586 (34.58\%), and 22,258 (34.07\%) cases were associated with EV71, CV-A16 and other enteroviruses, respectively (Fig 5 A). EV71 was the predominant serotype for mild cases in 2009, 2011, 2013, and 2015. CV-A16 was the predominant serotype among mild cases in 2010, 2012 and 2014. However, since 2016, other enteroviruses occupied the largest proportion among mild cases annually (Fig $5 \mathrm{~B}$ ). Most severe cases were caused by EV71. However, other enteroviruses have accounted for an increasing proportion of severe cases since 2013. In 2019, more than half of severe cases were associated with other enteroviruses (Fig 5 C). EV71 was the major pathogen among fatal cases. However, other enteroviruses and CV-A16 were the predominant serotypes among fatal cases in 2017 and 2019, respectively (Fig 5 D).

\section{Changes in HFMD epidemiology before and after EV71 vaccine introduction}

Compared to the pre-EV71 vaccine introduction period (2013-2015), there was no significant change in the gender composition among HFMD cases in the post-EV71 vaccine introduction period (2017-2019). The median age (2.5 years, IQR: 1.6-4.0) of cases during the post-vaccine period was significantly higher than that $(2.3$ months, IQR: $1.5,3.6)$ of cases during the pre-vaccine period. The number of cases decreased evidently among the age groups of under 3 years. All four regions of the province had an increase in the number of cases. More evident increasing trends were observed in the southern, northeastern, and northwestern areas. However, severe and fatal cases showed a significant decrease during the post-vaccine period. The proportion of HFMD cases caused by non-EV71/non-CV-A16 enteroviruses increased significantly. EV71-associated cases sharply decreased during the post-vaccine period (Table no. 2).

Table 2

Comparison of epidemiological characteristics between before (2013-2015) and after (2017-2019) introduction of EV71 vaccines in Yunnan Province 


\begin{tabular}{|c|c|c|c|}
\hline Items & $\begin{array}{l}\text { Pre-vaccine introduction } \\
(2013-2015)\end{array}$ & $\begin{array}{l}\text { Post-vaccine introduction } \\
(2017-2019)\end{array}$ & $\begin{array}{l}X^{2} \text { test } \\
P \text { value }\end{array}$ \\
\hline & $n=224246$ & $n=277731$ & \\
\hline Sex & & & 0.09 \\
\hline Female & $93731(41.80)$ & 116755 (42.04) & \\
\hline Male & $130515(58.20)$ & $160976(57.96)$ & \\
\hline Age & & & $<0.01$ \\
\hline $0-0.5$ & $2831(1.26)$ & $2909(1.05)$ & \\
\hline $0.6-2.9$ & $134097(59.80)$ & $157810(56.82)$ & \\
\hline $3-6.9$ & 78990 (35.22) & 104597 (37.66) & \\
\hline $7-14.9$ & 7655 (3.41) & $11223(4.04)$ & \\
\hline$\geq 15$ & $673(0.30)$ & $1192(0.43)$ & \\
\hline Median (IQR) & $2.3(1.5,3.6)$ & $2.5(1.6,4.0)$ & $<0.01^{a}$ \\
\hline Area & & & $<0.01$ \\
\hline Central & 95185 (42.45) & $104454(37.61)$ & \\
\hline Northeastern & 22449 (10.01) & 33311 (11.99) & \\
\hline Northwestern & 49057 (21.88) & $62432(22.48)$ & \\
\hline Southern & 57555 (25.67) & 77534 (27.92) & \\
\hline Case classification & & & $<0.01$ \\
\hline Mild & 220184 (98.19) & 275995 (99.37) & \\
\hline Severe & $4026(1.80)$ & $1724(0.62)$ & \\
\hline Death & $36(0.02)$ & $12(0.01)$ & \\
\hline Pathogen & & & $<0.01$ \\
\hline EV71 & $8642(44.34)$ & 3239 (14.41) & \\
\hline CV-A16 & 6078 (31.19) & $6307(28.06)$ & \\
\hline Others & 4769 (24.47) & 12929 (57.53) & \\
\hline 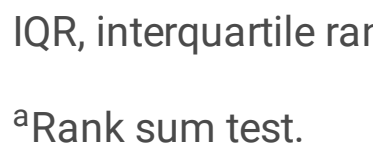 & & & \\
\hline
\end{tabular}




\section{Discussion}

The cumulative EV71 vaccination coverage rate reached $68 \%$ in Yunnan during the last four years. However, as estimated from the Chengdu study, to build herd immunity for ending EV71-related HFMD transmission, the vaccination coverage levels should reach $94.0 \%$ among infants [7]. Parental knowledge of the vaccine, adverse effects and cost were the most important factors that influenced willingness for EV71 vaccination uptake [8-10]. Yunnan is one of the undeveloped provinces in both the economy and culture in China [6]. To expand EV71 vaccination, more efforts on these influencing factors should be made by the local government.

The EV71 virus is divided into four genotypes (A, B, C and D) and is further divided into 12 subgenotypes. The current inactivated vaccine was derived from the $\mathrm{C} 4$ viral strain. The cross-protection effect of vaccines against various virus strains has not been clearly identified [11]. In a previous study, all of the EV71 isolates from Yunnan belonged to the C4 genotype $[12,13]$. The vaccine was highly matched with the predominant viral strains in the province. However, Yunnan Province is a border province of China and neighbors Southeast Asian countries, all of which are HFMD-endemic areas. Annual large cross-border population movement creates great challenges for preventing the transmission of infectious diseases in the province. HFMD was the most common gastrointestinal infection among imported cases in the border area of Yunnan [14]. EV71 genotypes C4, C2 and B5 have been demonstrated to cocirculate in Thailand and Vietnam $[15,16]$. Hence, monitoring changes in EV71 epidemic strains in the border area of the province is necessary to prevent the cross-border spread of these novel viral strains.

The present study showed ongoing epidemiological trends in HFMD since the disease was involved in NSNIDP in 2008. Following the introduction of EV71 vaccines in the province, case severity and fatality rates have been reduced, while the incidence rate has evidently increased. Similar findings have been reported in Chengdu and Guangzhou cities $[7,17]$. Virological surveillance indicated that non-EV71/nonCV-A16 enteroviruses contributed to the increase in incidence rate. However, the decrease in case severity and fatality was primarily attributed to the EV71 virus. The evidence strongly supported the beneficial impact of EV71 vaccination on the control of severe HFMD. However, the proportion of severe and fatal cases caused by other non-EV71 viruses had a significant increasing trend in Yunnan during the period of post-EV71 vaccine introduction. Thus, non-EV71 virus-associated HFMD should be highlighted to understand clinical manifestations to avoid disease progression.

Since 2013, the proportion of non-EV71/non-CV-A16 enteroviruses rose markedly, and it was greater than 50\% after 2017 in Yunnan. However, because most non-EV71/non-CV-A16 enteroviruses were not identified for specific serotypes, the current predominant viral strain was not well understood in the province. The whole etiological spectrum following EV71 vaccination in Xiangyang revealed that CV-A6 was the predominant serotype, followed by CV-A16, CV-A10, CV-A5, CV-A2 and EV71 [18]. However, since 2012 or even earlier, CV-A6 and CV-A10 have been the predominant enterovirus serotypes, overtaking EV71 and CV-A16 causing mild, severe and fatal cases of HFMD in some areas in China [19-21]. Moreover, an active virological investigation prior to the introduction of the EV71 vaccine in Yunnan 
showed CV-A6, CV-A10 and echovirus (E)-9 were the three most frequent serotypes among non-EV71/nonCV-A16 enteroviruses. CV-B5, CV-A9, E-30, E-18, CV-A4, C-B3, CV-A2 CV-A8, CV-A14, E-14, E-11, and CV-B4 were also detected [12]. These findings may indicate that current circulating non-EV71/non-CV-A16 serotypes can occur independently and may not be a result of EV71 vaccine selective pressure. Thus, long-term and expanding active etiological surveillance of HFMD is needed to understand the effect of EV71 vaccination on HFMD epidemiology and to reveal the existence of emerging viruses.

The seasonal pattern of HFMD presented yearly double peaks in Yunnan from 2005 to 2019. The two peak patterns were not changed by the introduction of the EN71 vaccine. Climatological factors including temperature and humidity, can induce EV71 and CV-A16 to cause differing epidemic scales [22]. Lager scales of the autumn peaks were detected in 2018 and 2019 in our study. Whether the changes in seasonal peaks are attributed to the changing serotype distribution combined with climate interruption needs to be investigated in further studies.

HFMD infections showed an increasing trend among all age groups after the introduction of EV71 vaccines in Yunnan. This finding was similar to the observation in Chengdu, which may be attributed to the significant increase in circulating non-EV71 enteroviruses [7]. The median age of HFMD infection was slightly delayed in our study. The Guangzhou study showed significant protection from the EV71 vaccine for HFMD among children 3 years of age [17]. Whether the delayed infection age was caused by the impact of EV71 vaccination or circulating non-EV71 enteroviruses, investigation on age-specific incidence by viral serotypes and vaccination rate should be carried out in further studies.

The main limitation of this study was that the EV71 vaccine is voluntary and self-paid in China. The demographics of children who were vaccinated were not recorded in the CDC vaccination surveillance system. Thus, we could not estimate the age-specific vaccination coverage rate. In this study, the vaccination coverage rate was estimated as the proportion of EV71 vaccinated people among the population of newborn children, which overstated the vaccination coverage rate and affected the full determination of the influence of EV71 vaccines on HFMD in Yunnan.

\section{Conclusion}

In conclusion, ongoing epidemiological changes in HFMD following the introduction of the EV71 vaccine were observed in Yunnan. EV71 vaccination helped to reduce severe HFMD. However, non-EV71/non-CVA16 enteroviruses became the predominant strain and brought larger scale incidence. Expanding EV71 vaccination and strengthening laboratory-based surveillance could further decrease the burden of severe HFMD and reveal the existence of emerging enteroviruses.

\section{Abbreviations}

CV-A16: coxsackievirus A16; E-9: Echo virus-9, EV71: enterovirus 71; HFMD: hand, foot, and mouth disease; NSNIDP: National Surveillance of Notifiable Infectious Disease Programme; IPIMS: 


\section{Declarations}

\section{Ethical approval and consent to participate}

The Yunnan Provincial Center for Disease Control and Prevention ethics committee granted ethical approval for the study and used de-identified surveillance data.

\section{Consent for publication}

Not applicable.

\section{Availability of data and materials}

The datasets used and analyzed during the current study are available from the corresponding author on reasonable request.

\section{Competing interests}

The authors report no conflicts of interest.

\section{Funding}

This research received the grants from funding of the high-quality professional youth introduction project of Kunming City when performing data analysis, manuscript writing, and interpretation of data, and funding of Yunnan health training project of high level talents (no. D-2017053), Top experts training project for the academy and technology in Kunming City and Yunnan province (202005AC160066), and Top young experts project in Kunming(C201914013) when performing study design.

\section{Authors' contributions}

LJ was responsible for overall data management, analysis and wrote the paper. TH designed the study. And $\mathrm{HJ}, \mathrm{XT}, \mathrm{XX}$ conceived the study. All authors reviewed and approved the final manuscript.

\section{Acknowledgments}

The authors thank all the staffs of the Yunnan CDCs and hospitals who participated in data collection. 


\section{References}

1. Zhuang ZC, Kou ZQ, Bai YJ, Cong X, Wang LH, Li C, et al. Epidemiological Research on Hand, Foot, and Mouth Disease in Mainland China. Viruses. 2015; 7(12):6400-6411.

2. Yang B, Liu F, Liao Q, Wu P, Chang Z, Huang J, et al. Epidemiology of hand, foot and mouth disease in China, 2008 to 2015 prior to the introduction of EV-A71 vaccine. Euro Surveill. 2017; 22(50):1-10.

3. Zhu FC, Meng FY, Li JX, Li XL, Mao QY, Tao H, et al. Efficacy, safety, and immunology of an inactivated alum-adjuvant enterovirus 71 vaccine in children in China: a multicentre, randomised, double-blind, placebo-controlled, phase 3 trial. Lancet. 2013; 381(9882):2024-2032.

4. Zhu F, Xu W, Xia J, Liang Z, Liu Y, Zhang X, et al. Efficacy, safety, and immunogenicity of an enterovirus 71 vaccine in China. N Engl J Med. 2014; 370(9):818-828.

5. Li R, Liu L, Mo Z, Wang X, Xia J, Liang Z, et al. An inactivated enterovirus 71 vaccine in healthy children. N Engl J Med. 2014; 370(9):829-837.

6. Xu W, Jiang L, Thammawijaya P, Thamthitiwat S. Hand, foot and mouth disease in Yunnan Province, China, 2008-2010. Asia Pac J Public Health. 2015; 27(2):NP769-777.

7. Han Y, Chen Z, Zheng K, Li X, Kong J, Duan X, et al. Epidemiology of Hand, Foot, and Mouth Disease Before and After the Introduction of Enterovirus 71 Vaccines in Chengdu, China, 2009-2018. Pediatr Infect Dis J. 2020; 39(10):969-978.

8. Li T, Wang H, Lu Y, Li Q, Chen C, Wang D, et al. Willingness and influential factors of parents to vaccinate their children with novel inactivated enterovirus 71 vaccines in Guangzhou, China. Vaccine. 2018; 36(26):3772-3778.

9. Qi L, Su K, Xia Y, Tang W, Shen T, Li Q. Enterovirus 71 vaccine acceptance among parents of children $<5$ years old and their knowledge of hand, foot and mouth disease, Chongqing, China, 2017. PLoS One. 2019; 14(11):e0225569.

10. Wang Y, Meng F, Li J, Li G, Hu J, Cao J, et al. Willingness of parents to vaccinate their 6-60-month-old children with EV71 vaccines: a cross-sectional study in rural areas of northern Jiangsu Province. Hum Vaccin Immunother. 2020; 16(7):1579-1585.

11. Liang Z, Wang J. EV71 vaccine, an invaluable gift for children. Clin Transl Immunology. 2014; $3(10): \mathrm{e} 28$.

12. Du Z, Zhao Y, Luo Y, Du L, Gan Q, Zhang H, et al. Ongoing change of severe hand, foot, and mouth disease pathogens in Yunnan, China, 2012 to 2016. J Med Virol. 2019; 91(5):881-885.

13. Wang Y, Li Y, Yang Y, Peng C, Fu X, Gu X, et al. Virological investigation of genetic variation of enterovirus type 71 in hand, foot and mouth disease. Exp Ther Med. 2020; 20(1):543-549.

14. Jiang L, Huang T. Comparison of the epidemiological aspects of acute infectious diseases between foreign and native imported cases in the border counties of Southwest China, 2008-2017. Epidemiol Infect. 2019; 147:e230.

15. Noisumdaeng P, Sangsiriwut K, Prasertsopon J, Klinmalai C, Payungporn S, Mungaomklang A, et al. Complete genome analysis demonstrates multiple introductions of enterovirus 71 and 
coxsackievirus A16 recombinant strains into Thailand during the past decade. Emerg Microbes Infect. 2018; 7(1):214.

16. Le TV, Nguyen VTT, Nguyen QH, Pham DT. Molecular epidemiology analysis of enterovirus 71 strains isolated in Dak Lak, Vietnam, 2011-2016. J Med Virol. 2019; 91(1):56-64.

17. Du Z, Huang Y, Bloom MS, Zhang Z, Yang Z, Lu J, et al. Assessing the vaccine effectiveness for hand, foot, and mouth disease in Guangzhou, China: a time-series analysis. Hum Vaccin Immunother. $2021 ; 17(1): 217-223$.

18. Meng XD, Tong Y, Wei ZN, Wang L, Mai JY, Wu Y, et al. Epidemical and etiological study on hand, foot and mouth disease following EV-A71 vaccination in Xiangyang, China. Sci Rep. 2020; 10(1):20909.

19. Yang Q, Ding J, Cao J, Huang Q, Hong C, Yang B. Epidemiological and etiological characteristics of hand, foot, and mouth disease in Wuhan, China from 2012 to 2013: outbreaks of coxsackieviruses A10. J Med Virol. 2015; 87(6):954-960.

20. Weng Y, Chen W, He W, Huang M, Zhu Y, Yan Y. Serotyping and Genetic Characterization of Hand, Foot, and Mouth Disease (HFMD)-Associated Enteroviruses of No-EV71 and Non-CVA16 Circulating in Fujian, China, 2011-2015. Med Sci Monit. 2017; 23:2508-2518.

21. Wang J, Teng Z, Cui X, Li C, Pan H, Zheng Y, et al. Epidemiological and serological surveillance of hand-foot-and-mouth disease in Shanghai, China, 2012-2016. Emerg Microbes Infect. 2018; 7(1):8.

22. Du Z, Huang Y, Lawrence WR, Xu J, Yang Z, Lu J, et al. Leading Enterovirus Genotypes Causing Hand, Foot, and Mouth Disease in Guangzhou, China: Relationship with Climate and Vaccination against EV71. Int J Environ Res Public Health. 2021; 18(1):292.

\section{Figures}
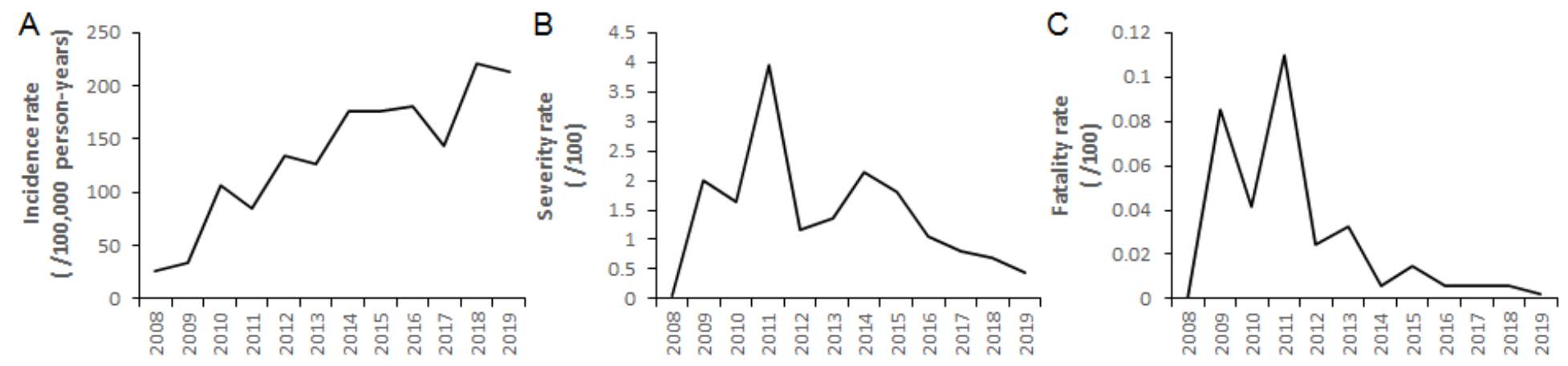

\section{Figure 1}

Annual incidence rates (per 100000 population), severity rates (per 100 cases), and fatality rates (per 100 cases) of reported hand, foot and mouth disease patients in Yunnan Province 2008-2019. 

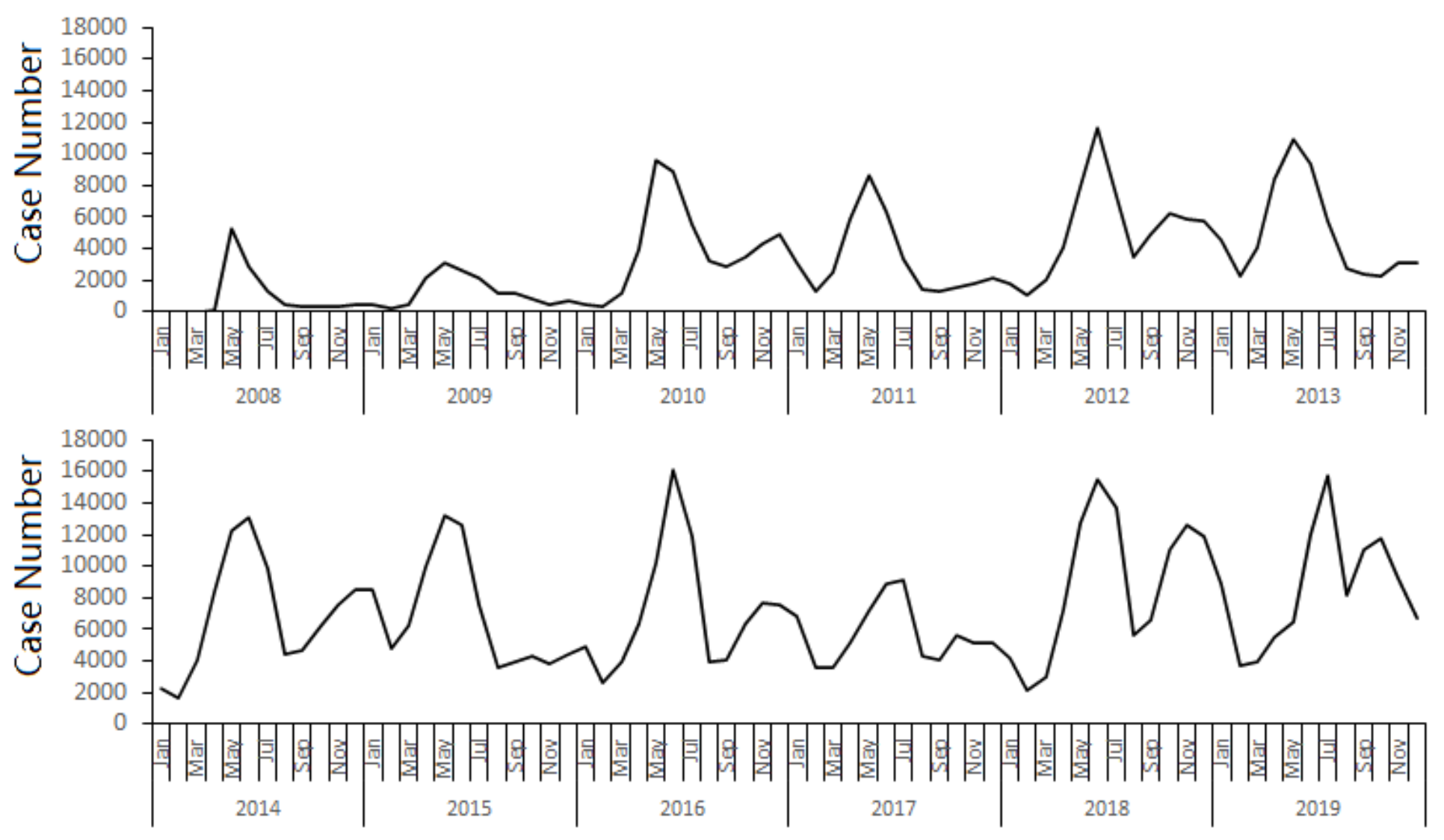

\section{Figure 2}

Reported cases of hand, foot and mouth disease in Yunnan Province by month 2008-2019.
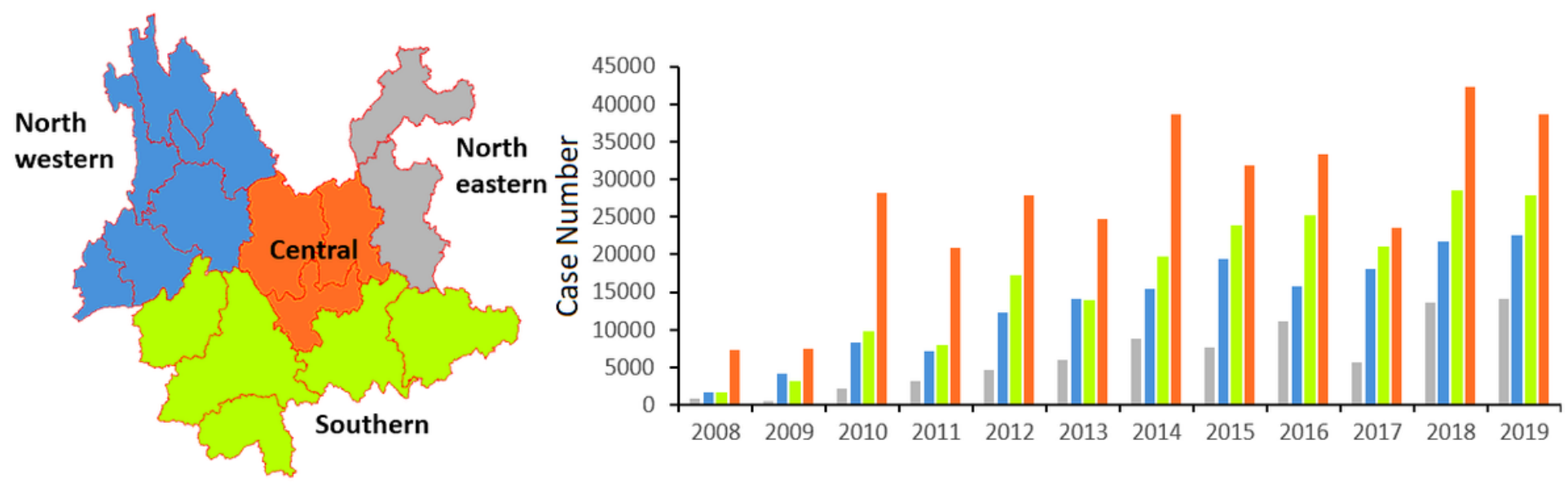

Figure 3

Reported cases of hand, foot and mouth disease in Yunnan Province by area 2008-2019. Note: The designations employed and the presentation of the material on this map do not imply the expression of any opinion whatsoever on the part of Research Square concerning the legal status of any country, territory, city or area or of its authorities, or concerning the delimitation of its frontiers or boundaries. This map has been provided by the authors. 
A
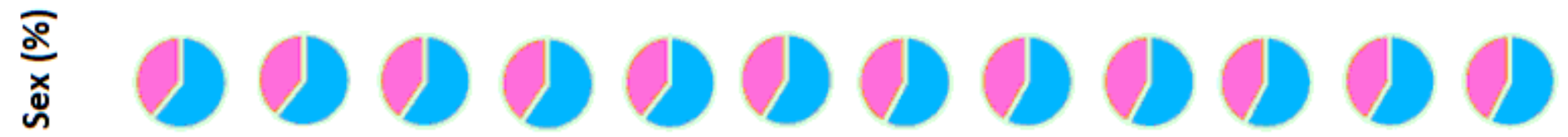

B

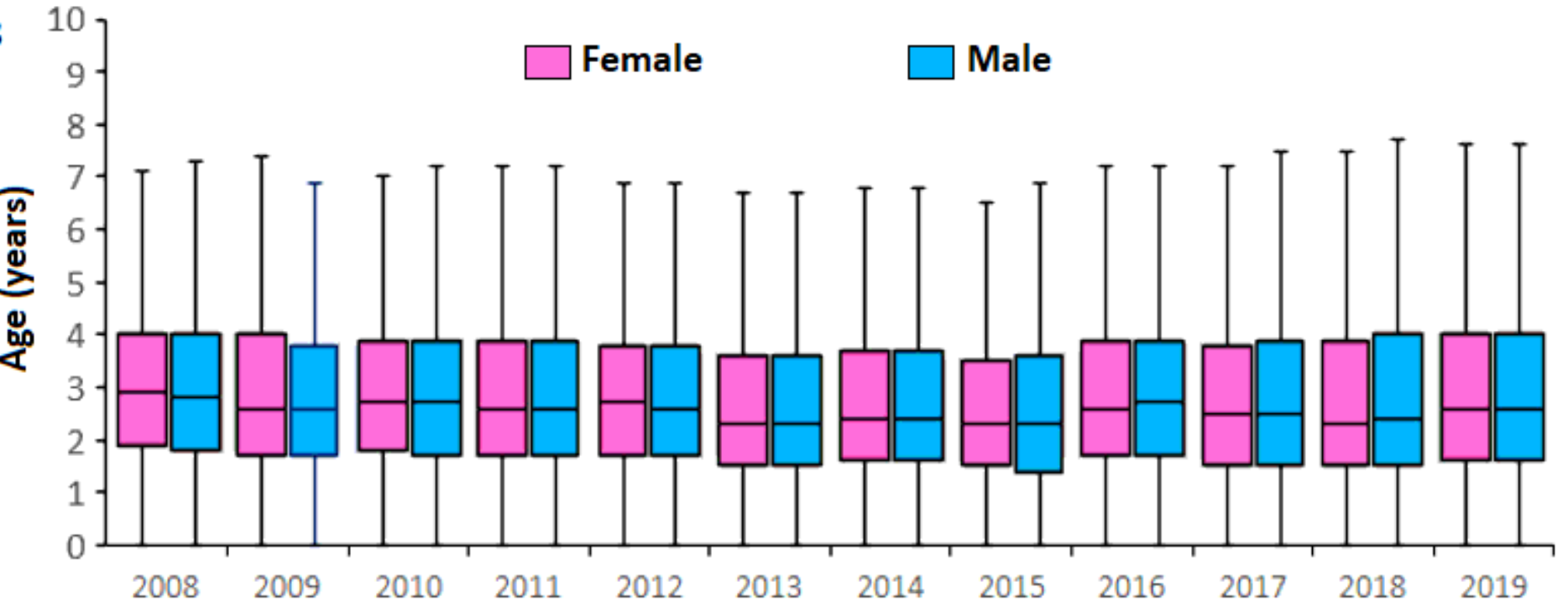

Figure 4

Sex composition and age distribution of reported hand, foot and mouth disease patients in Yunnan Province by year 2008-2019. 


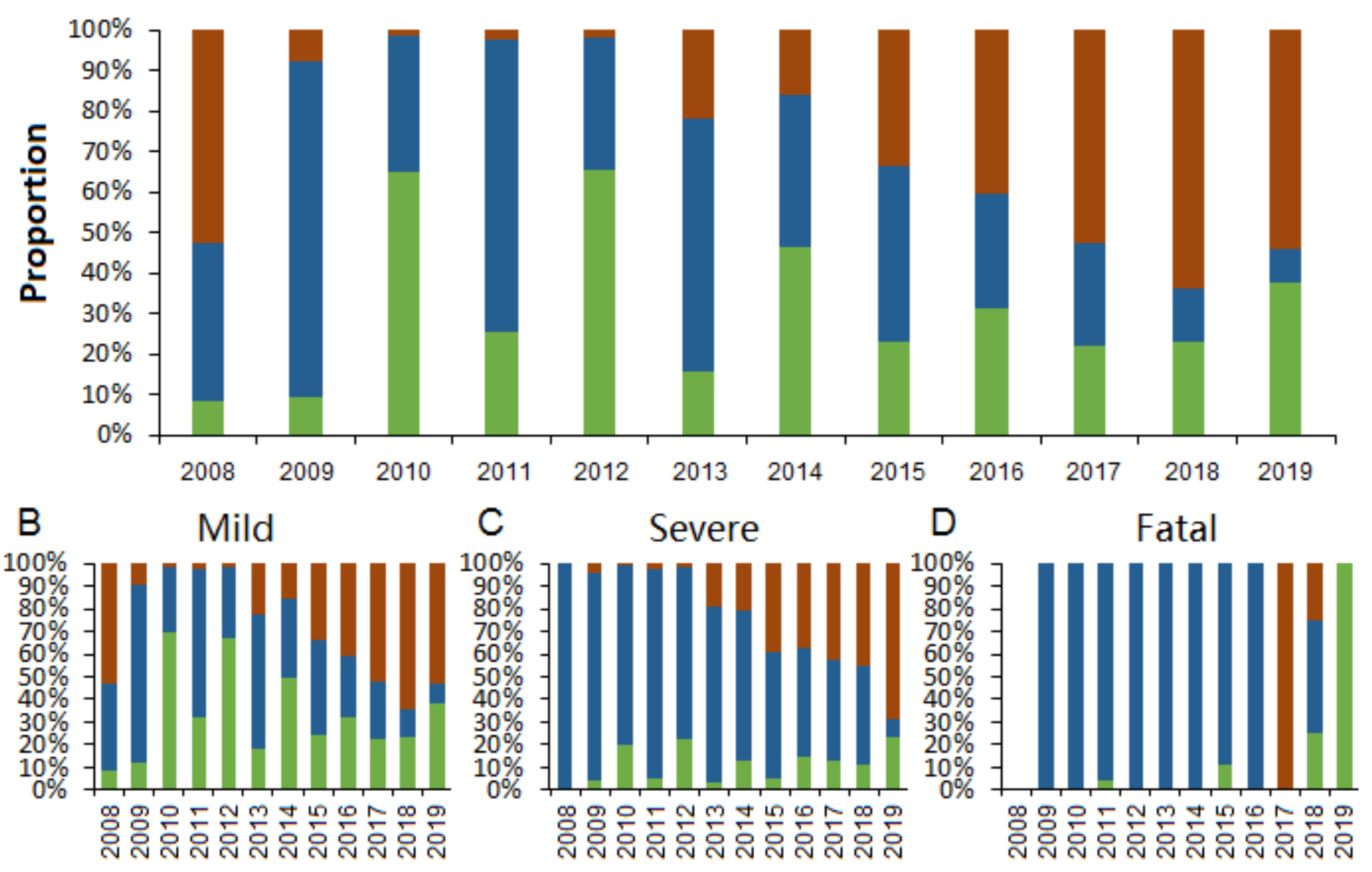

घCV-A16 — EV71 — Other enteroviruses

\section{Figure 5}

Proportions of enterovirus serotypes among laboratory-confirmed, mild, severe, and fatal cases of hand, foot, and mouth disease in Yunnan Province by year 2008-2019. 